\title{
THE ASSOCIATED FRIENDS
}

\section{New Member}

With the addition of one new member whose name is listed below, the membership of the Associated Friends stands at $38 \mathrm{I}$.

Robert G. Dickson

\section{Gifts}

The Library has received gifts from the following persons:

Charles S. Aitkin, '8o

Barnet J. Beyer

Edmond W. Billetdoux

Moses J. Brines

G. Harold Buttler, Jr., '36

Robert C. Clothier

Mrs. Drury W. Cooper

William D. Danberry, '22

Hiram E. Deats

William H. S. Demarest, ' 83

Charles D. Deshler, Jr., ' 85

Nelson Dunham

Dr. and Mrs. Wells P. Eagleton

Clayton M. Hall

John A. Hayes

Albert W. Holzmann, 'I 7

Rudolf Kirk

I. Robert Kriendler, ' 36

J. Carl Kriendler

William H. F. Lamont

Robert B. Littell, '95

Albert E. Meder, Jr.

Richard Morris, '99

Edna M. Netter

Roy F. Nichols, 'I 8
Sydney P. Noe, 'Io

Mrs. Margaret E. O'Neill

George A. Osborn, '97

Victor H. Paltsits

Remigio U. Pane, ' 38

Clarence E. Partch

Herman A. Plusch, 'o4

Carroll C. Pratt

Charles W. Stevens, Jr., 'O2

John L. Stillwell, '79

Frank H. Stobaeus, 'o8

Agnes W. Storer

Hobart E. Studley, ' 93

Charles T. Thompson, 'o8

Peter A. Van der Meulen

Ralph D. Van Duzer, 'o7

Oscar M. Voorhees, '88

Selman A. Waksman, 'I 5

John P. Wall

Harry B. Weiss

Percival Wilde

Helen L. Williamson

Julia B. Williamson

Nicholas Williamson, '02

Ralph G. Wright 